\title{
Design of a Radial Turbine for Wastegated Turbocharger
}

\author{
Hanqin Yang*, Qingbin Li, Liaoping Hu, Xiong Meng, Guangqing He, Lin Liu
}

Technology Center, Hunan Tyen Machinery Co., Ltd. Hengyang, China

Email address:

dijiaqiu@foxmail.com (Hanqin Yang)

${ }^{*}$ Corresponding author

\section{To cite this article:}

Hanqin Yang, Qingbin Li, Liaoping Hu, Xiong Meng, Guangqing He, Lin Liu. Design of a Radial Turbine for Wastegated Turbocharger. International Journal of Fluid Mechanics \& Thermal Sciences. Special Issue: Fluid Mechanics \& Thermal Sciences in Turbomachines. Vol. 6, No. 1, 2020, pp. 19-26. doi: 10.11648/j.ijfmts.20200601.13

Received: January 28, 2020; Accepted: February 21, 2020; Published: February 28, 2020

\begin{abstract}
With the increasing popularity of turbocharging technology in the automotive industry, and people's increasing awareness of environmental protection, engineers are paying more attention to the technical development of turbochargers, while more advanced tools and methods are being used in the actual turbocharged design process to improve the accuracy and efficiency of research and design. Traditional turbocharger design methods are often based on experimental data and empirical models, and long development cycles conflict with market demand, and high performance conflict with high reliability. In this paper, in order to resolve this significant contradiction, a design process based on a variety of CAE methods and tools is proposed for the design of turbines, which are key components of turbochargers. This process was early applied to the design of turbocharger compressor, and then under the original boundary conditions, considering the challenges of high temperature and high engine demand, it has been improved and applied to the design of a 9L off-road country Tier-IV engine turbine in the Chinese market. The analysis shows that the turbine has good aerodynamic performance and structural reliability. The turbine performance was tested in gas stand the also verified on the engine test. This study demonstrated that the design was successful and future new turbine design can use similar procedure.
\end{abstract}

Keywords: Turbine, Turbocharger, Tier-IV, CAE, CFD

\section{Introduction}

Efforts to achieve more power with less fuel consumption dated all the way back to the very start of engine design. In recent years, the engine market becomes more competitive. The increasing awareness of energy conservation and environmental protection have demanded engine manufacturing to use better turbocharger system to meet the needs of customers and regulations. Researchers have become increasingly demanding in terms of performance and reliability of turbochargers. The design of turbines and compressors has faced huge challenges. In the process of turbocharger design, enterprises and designers are often under the pressure of rapid market updates and iterations. This article mainly describes a turbine design method for the off-road Tier IV engine market, which involves the aerodynamic design, reliability analysis, engine matching, and test verification of the turbine. The simplest shape of a turbocharger is wastegate turbocharger. Wastegate turbochargers have widely applications due to the reliability and easy to control. The turbochargers trend to reduce the size. The turbine or compressor wheel rotate more quickly with increasing exhaust gas volume as the engine speed increases. The increase in speed is accompanied by increasing mechanical and thermal load on the components involved. This brings more challenges not only in aerodynamic design but also in reliability mechanical design.

Generally speaking, the turbines of traditional turbochargers can be divided into radial flow, mixed flow and axial flow turbines. Among them, the turbochargers for vehicles mostly use radial or mixed flow turbines. The design methods of radial flow turbines have been studied for a long time [1]. The classical turbine design is based on one-dimensional theoretical calculations. Some of the turbine-related thermodynamic formulas and velocity triangles are still used now by researchers and engineers to predict and evaluate performance. The development of computer-aided technology Baines and Wallace [2] proposed a numerical turbine design method, introduced a 
quasi-three-dimensional flow field analysis. A large number of optimizations based on experimental tests used computational simulation are used recently. With the increase of the computer CPU speed, the analytical time has shorten significantly. In the past ten years, with the advance of the intelligent manufacturing, the more complicated turbine geometry can be built. The performance and reliability of turbochargers have a substantial improvement.

With the development of numerical methods, a faster, better, and time economic turbomachinery design methods were proposed based on an empirical database combined with computer-aided design. This method is used in turbomachinery engineering applications. It is of great significance in market competition. In this turbine design, the turbine design follow the turbomachinery processors developed recently [3]. The method of combining empirical data with computational assistance is used for turbine design. This method can respond quickly and efficiently to meet market demands and create more economic value for the enterprise. The design of the turbine has become a systematic task. The focus of the turbine design was on the performance in the past. Now the turbine design has become more attention to on engine performance improvement in whole operating range, i.e. the performance of both the engine design point and the non-design point.

\section{Turbine Design Process}

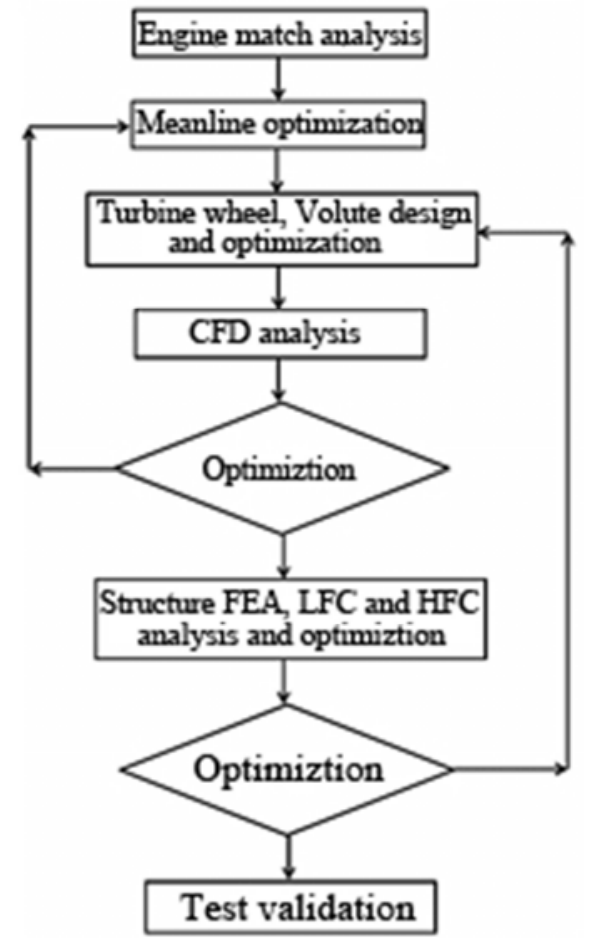

Figure 1. Turbine design process.

With the development of simulation technology in recent years, turbine design methods have developed rapidly. In the turbine initial design, the performance goals of the turbine are mainly obtained through the engine simulation software. After set up the turbine design goals, one-dimensional parameter and the meridian streamline design are performed, and finally the three-dimensional fluid analysis is carried out for detailed optimizations as shown in Figure 1 [3-5]. The meridional streamline design can be obtained by direct design or scaling of the existing design. In this design, the initial turbine design is first obtained by searching the in-house turbine database to find a similar turbine to meet the design goal. This method can save a lot of time and cost. Through the comparison of different scaling turbines and further optimizations, the predicted turbine flow and efficiency are obtained, and the engine performance is checked again by the engine simulation to see whether turbine design can meet the design targets.

After the design of the meridian streamlines, more detail three-dimensional optimization is needed, such as thickness analysis, blade load, and off-design performance etc. This step mainly adjusts the turbine and turbine housing to achieve the highest efficiency at both the design point and off-design points. In the turbine design process, the turbine efficiency at the off-design point is also very important. The off-design performance can be further adjusted through the area of the tongue of the turbine housing. After the turbine was designed, the reliability analysis should perform. During the structural check phase, the structural strength and frequency of the turbine are calculated. If reliability goal cannot be meet, the turbine need to be adjusted to meet the reliability requirements. These changes often affect the aerodynamic performance. Therefore, the aerodynamic calculations of the turbine are also required to verify the performance after the structure adjustments. The final turbine design should meet both performance and reliability goals.

\section{Engine Matching}

For a 9 liter off-road Tier IV engine, the turbocharged intercooler and SCR technology are adopted. The specific structural parameters of the engine are as follows:

Table 1. Engine parameters.

\begin{tabular}{ll}
\hline Items & Parameters \\
\hline Engine type & Six-cylinder, four-stroke \\
Fuel type & Diesel \\
Number of valves & 4 \\
Bore diameter $\times$ Stroke length $(\mathrm{mm})$ & $126 \times 130$ \\
Displacement & $9.73 \mathrm{~L}$ \\
Calibration power & $130 \mathrm{kw}$ \\
Maximum Torque & $950 \mathrm{~N} . \mathrm{m}$ \\
\hline
\end{tabular}

The turbocharger engine model is shown in Figure 2. This model is based on the engine test data and the actual turbine gas-stand map. The intake environment conditions, intercooler conditions, intake pipe, injector, cylinder, crankcase, exhaust gas pipeline and exhaust environment are modeled with corresponding throttling modules. The intake and exhaust pipelines, intercoolers, intake and exhaust valves, cylinders, fuel injectors, crankcases, etc. are connected according to the actual structural size of the diesel engine. The module parameters are set. Among them, the intake and 
exhaust valve lift curve, fuel injection timing, and combustion model are directly input from the provided data. The combustion model uses the in-cylinder direct injection diesel Weber combustion model. The mechanical loss is performed using the Chen-Flynn empirical formula. Components such as air filter, exhaust gas after-treatment system, and muffler are simplified, and pressure loss elements are used in the model to calculate their impact on engine power performance. The intake and exhaust flow coefficients are calibrated by test parameters. Due to changes in cylinder pressure and air temperature, the main combustion period will be different. The intake air volume is adjusted by adjusting the valve timing $[6]$.

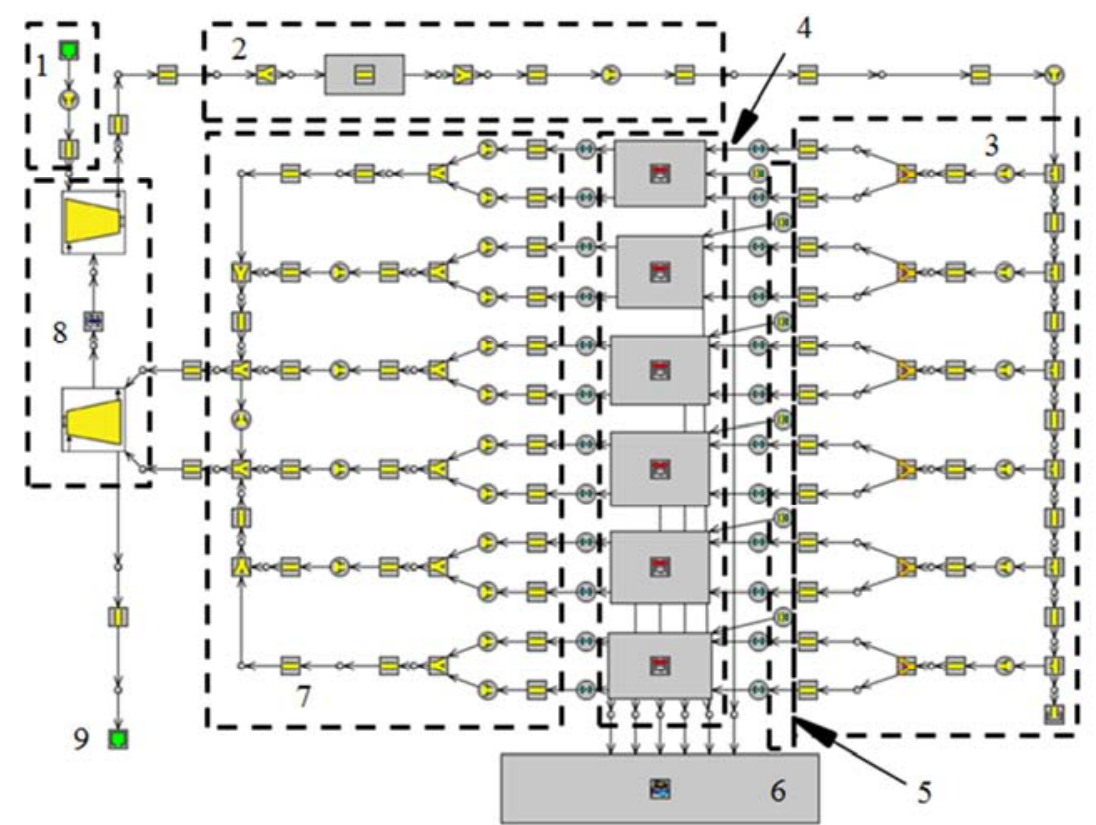

1. Intake environment 2. Intercooler 3. Intake manifold 4. Cylinder 5. Injector 6. Crankcase 7. Exhaust manifold 8. Turbocharger 9. Exhaust environment.

Figure 2. Turbocharged diesel engine model.

By calibrating the simulation model, an old turbine with engine test data and gas-stand data were input into engine simulation tool. The test data of the engine intake air flow is compared with the engine simulation calculations. As shown in Figure 3, the test data curve is in good agreement with the engine simulation data. The maximum error at high speed is less than $3 \%$, which indicates that the engine parameter setting is appropriate。

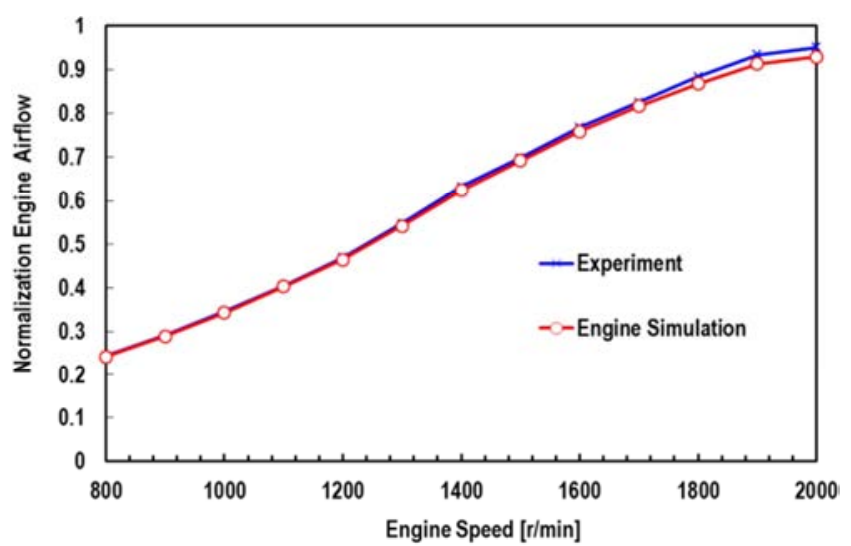

Figure 3. Comparisons between experiment data and simulation data.

Based on this engine simulation model, under the same torque characteristics, a comparative analysis of the most suitable turbocharger and the engine performance target is selected. The simulation results are shown in Figure 4. The existing turbocharger cannot reach the engine performance. The target, in particular, the specific fuel consumption at the calibration point is $4 \mathrm{~g} / \mathrm{kW}$. h higher than the performance target and the boost pressure is $10 \mathrm{kPa}$ lower. Existing compressors cannot meet the pressure requirements of the engine, and the intake air flow is also low. The power of the compressor is mainly provided by the turbine. The turbine flow characteristics, Figure $4 \mathrm{c}$ show that the original turbine cannot meet the performance targets.

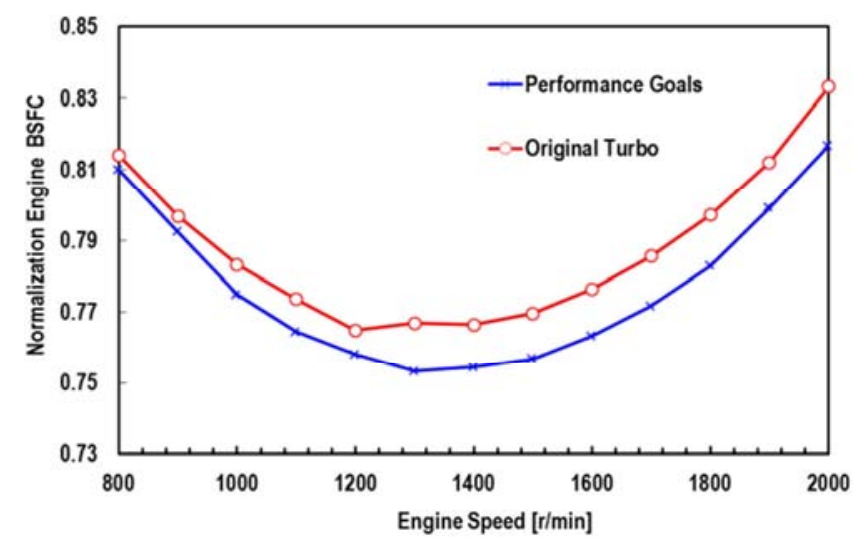

(a) engine specific fuel consumption. 


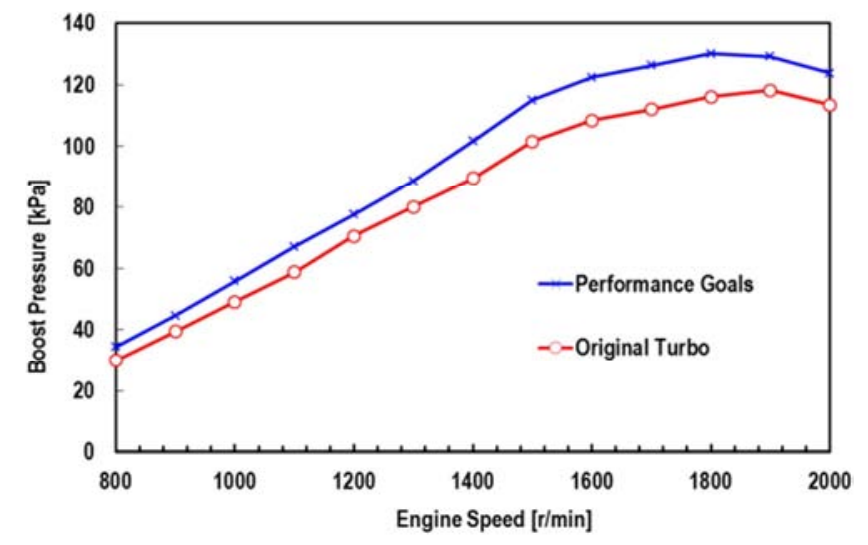

(b) engine boost pressure.

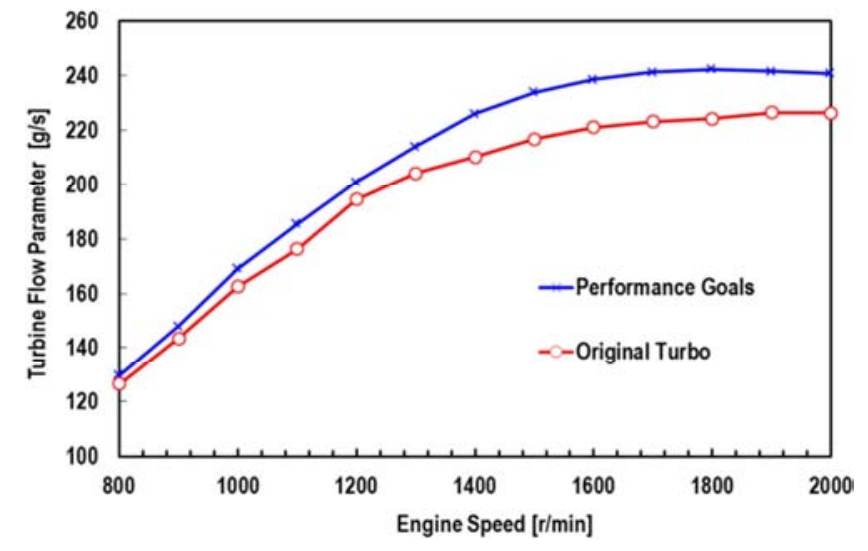

(c) turbine flow.

Figure 4. Engine Data Analysis.

Under low-speed operating conditions of the engine, the turbine has a small circulation capacity and high efficiency. At the same time, it is also supported by the exhaust pulse energy of the engine, and the turbine output shaft work. To meet the compressor's work requirements and meets the performance target, a low-speed turbine performance is very critical. The fuel consumption is less affected by the pumping loss at low engine speed. The existing turbine as shown in Figure 4 cannot meet both low speed and high speed performance targets. When the engine is at medium speed, the existing turbine efficiency is reduced, and the turbine output shaft power is weakened. The compressor's functional power can no longer meet the engine demand, and the boost pressure is reduced. When the engine is at high speed, the existing turbine efficiency is further reduced, and the gap between the supercharging capability and the performance target is widened. Due to the small turbine flow capacity, the exhaust back pressure of the engine is high. The pumping loss is further deteriorated and cause a great impact on the specific fuel consumption of the engine.

The existing turbochargers are unreasonably matched, especially the turbine's circulation capacity is $6 \%$ smaller cause engine high speed high back pressure. The turbine efficiency is low at the engine calibration point as well as off-design points. The new turbine needs to increase the flow capability and also needs to have high efficiency in all engine operating range. The efficiency characteristics of the new turbines are characterized by high low-speed efficiency and high-speed efficiency.

\section{Turbine Design}

In study, we perform the design of a new turbine wheel and optimizing turbine stage to meet the engine performance goals. Based on the engine simulation requirements, the design point is set at a high-speed calibration point. The design engine rotational speed is $2000 \mathrm{r} / \mathrm{min}$. The turbine stage performance targets are based on the engine simulation. The specific performance targets are shown in Table 2 .

Table 2. Turbine design information.

\begin{tabular}{ll}
\hline Items & Parameters \\
\hline Turbine maximum speed & $70000 \mathrm{r} / \mathrm{min}$ \\
Design expansion ratio & 2.0 \\
Maximum mass flow & $0.240 \mathrm{~kg} / \mathrm{s}$ \\
Efficiency requirements & as high as possible \\
\hline
\end{tabular}

According to the aerodynamic performance target, there are two design methods for the turbine. The first method is direct design based on one-dimension analysis. The main geometries of the turbine inlet and outlet are designed based on one-dimensional results. The second design method is to select an existing design which performance is close to target and scale it as a baseline to optimize or just optimize the turbine stage wheel and housing match. This method is used more in engineering applications and can obtain higher efficiency at short time. In this paper, the second method is used to scale the turbine to obtain a turbine wheel geometric model as a baseline wheel.

The mass flow rate is proportional to the square of the inlet diameter of the turbine, the ideal turbine is scaled. Based on an imported radial turbine with a diameter of $86 \mathrm{~mm}$, this turbine can achieve an adiabatic isentropic efficiency of more than $75 \%$ in practical applications, and the market application is relatively mature. The turbine scale factor can be obtained as

$$
\frac{M_{\text {scale }}}{M}=\frac{D_{\text {scale }}{ }^{2}}{D^{2}}=k
$$

According to the design goals of the turbine mass flow, no adjustments are made to the turbine wheel blade shape, the number of blades, the inlet geometry, the outlet geometry, the hub diameter, etc. The scaling factor $\mathrm{k}$ is the only parameter to be considered in this study. Three turbine with scale factors $\mathrm{k}$ $=0.865,0.9$, and 0.96 were constructed. CFD simulations were performed for all three turbine wheels with same turbine housing.

In order to simulate and analyze the performance of the three turbine stages, a CFD model (Figure 5) is established to perform the performance analysis. The calculation model includes an inlet section, a turbine box, a single-channel turbine, and an outlet section. The fluid domain uses a hexahedral structure mesh as shown in Figure 5. The Y+ value is set to be less than 3 , and the total number of grids is 2.73 
million cells. The commercial CFD software FINE / Turbo is used, the Shear stress Transport (SST) model is adopted to simulate the turbulent flow and the circumferential interface of the turbine rotor and the turbine housing are conservatively coupled by pitchwise interface. This connection method can ensure that the mass, momentum, and energy of the static subsurface are strictly conserved.

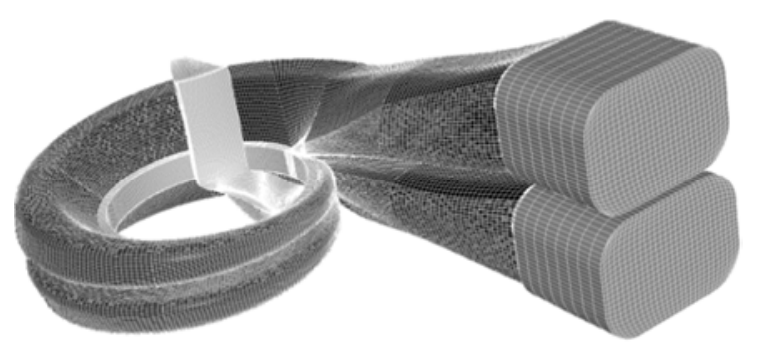

Figure 5. CFD Model.

The steady CFD analysis of the three turbine stages at

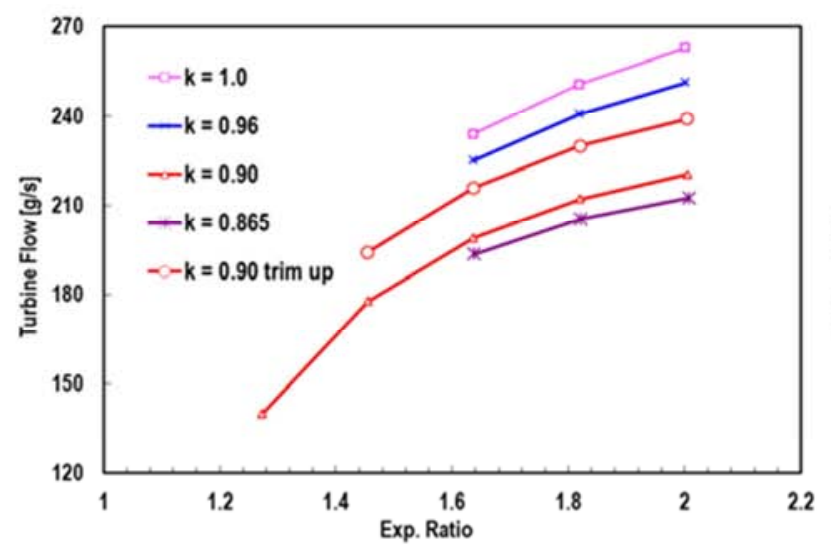

turbine wheel rotational speed of $70,000 \mathrm{r} / \mathrm{min}$ is performed and results are compared. The calculation results show that although the scale factor is proportional to the mass flow rate, but affected by the blade boundary layer, the increase of the tongue area of the turbine is not proportional to the scale factor $\mathrm{k}$. However, the smaller the scale factor $\mathrm{k}$, the smaller the change rate of the mass flow rate of the turbine. At the same time, considering the structural reliability of the turbine, hub diameter and blade thickness have some limitations. The scale factor $\mathrm{k}$ cannot be reduced all the time. At the same time, the efficiency of the turbine gradually decreases with the scaling factor $\mathrm{k}$. The smaller the scaling factor the lower the efficiency of the turbine. The main reason for this is that the percentages of the tip clearance in blade height of the turbine increase. In general, although the flow rate of the turbine has changed the shapes of the turbine flow curves and the efficiency curves have not changed, that is, the change trends are consistent as shown in Figure 6.

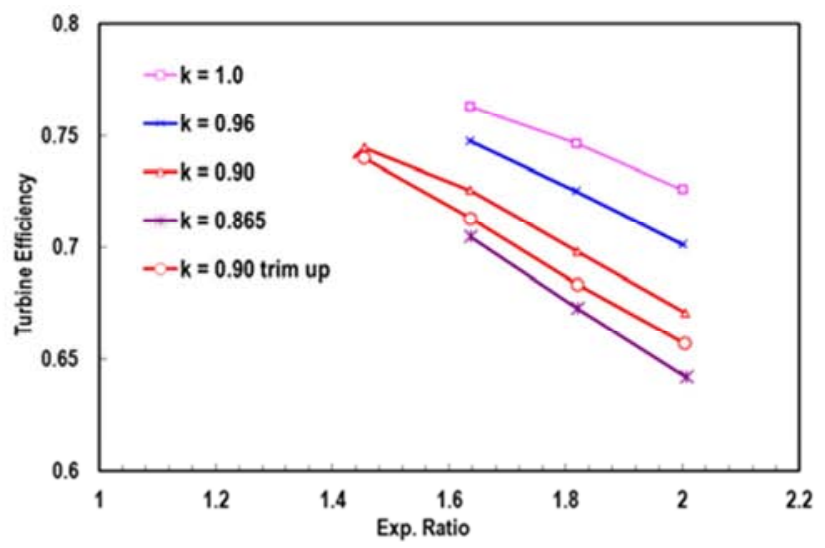

Figure 6. CFD Turbine Parameter.

\section{Turbine Aerodynamic Optimization}

Based on the CFD simulation results in the previous section 4 , the turbine performance results of $\mathrm{k}=0.9$ scale factor are closer to the performance targets for both flow parameter and efficiency. Based on this turbine geometry, the trim and blade size of the turbine are optimized to meet the aerodynamic performance goals. By adjusting the outlet size of the turbine, the simulation results $\mathrm{s}$

how that $(\mathrm{k}=0.9$ trim-up in figure 6$)$ the mass flow has reached the requirements, but the efficiency of the turbine has decreased, mainly due to the change of the mass flow rate inside of the turbine blades, the loading changes and the secondary flow loss near shroud increased. Therefore, the efficiency of the turbine needs to be improved by optimizing it.

Because the turbine operates in the entire engine speed range, it is necessary to improve the turbine efficiency in the entire engine operating range, including the efficiency at off-design points. The turbine needs to match with the compressor to obtain the best performance. The turbine performance is usually characterized by the ratio of the turbine inlet tip speed to the isentropic absolute speed of the outlet, that is, the speed ratio BSR [7]:

$$
\text { BSR }=\frac{U}{C_{i s}}=\frac{U}{\sqrt{2 C_{p} T_{0}\left[1-\pi^{\frac{1-k}{k}}\right]}}
$$

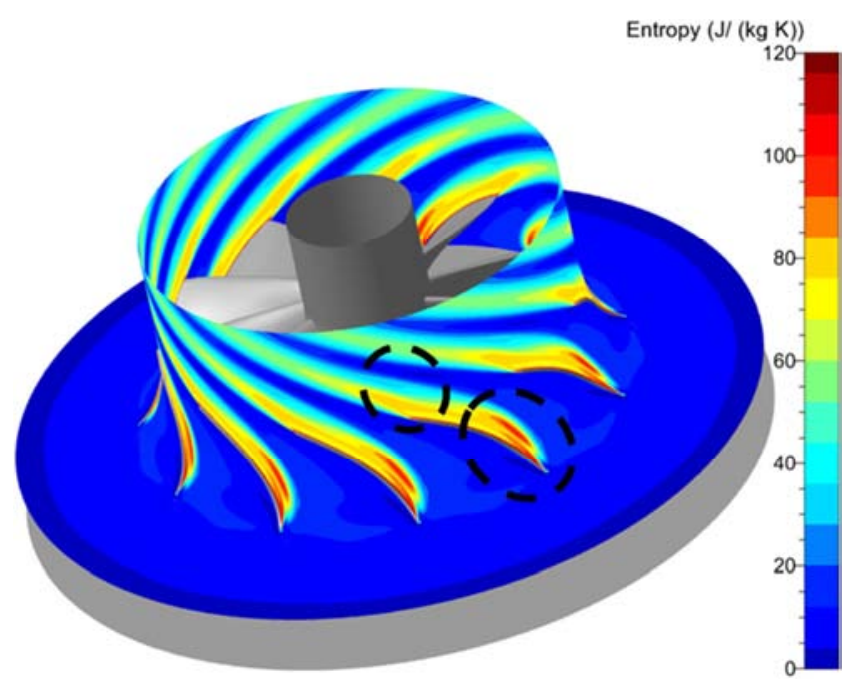

(a) Baseline. 


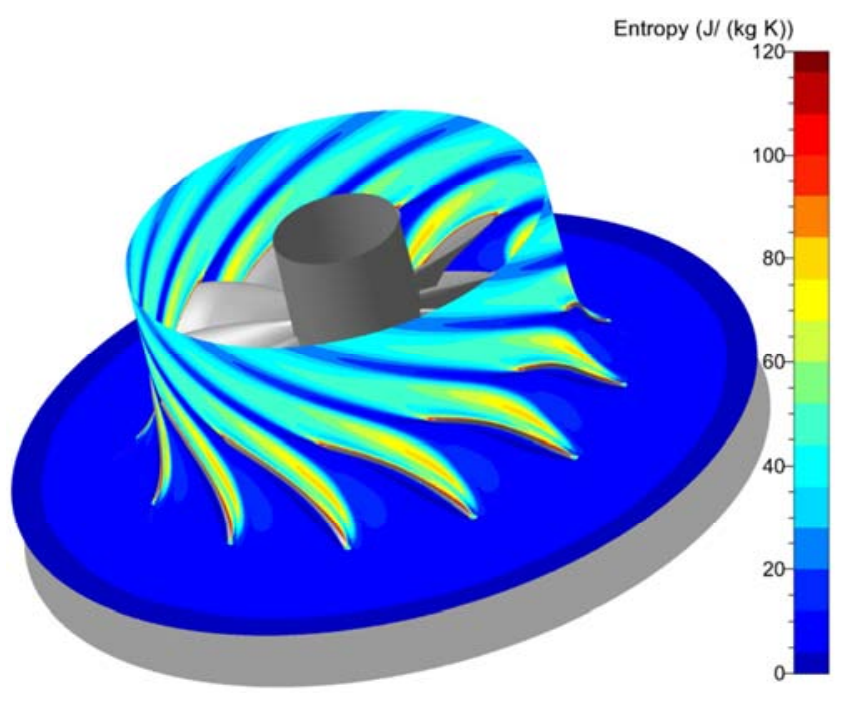

(b) Optimization.

Figure 7. Entropy at $96 \%$ span at BSR=0.7 near peak efficiency.

When the turbine matches to $\mathrm{BSR}=0.7$, the turbine efficiency is close to the peak. The entropy diagram of the blade cross section near the shroud is compared as shown in Figure 7. At the shroud of the original turbine (a), part of the fluid crosses the blade due to the tip clearance [8]. Especially at the exit position of the blade, this flow becomes more and more severe as the ratio of the clearance to the height of the blade becomes larger.

The tip flow is mixed with the mainstream and finally mixed with the wake flow for original design. The current scaled turbine got optimization for the turbine blade. After the blade shape at the shroud is optimized and adjusted, the entropy increase area on the suction surface of the turbine blade is significantly reduced, the flow field tends to be stable, and the overall entropy value decreases.

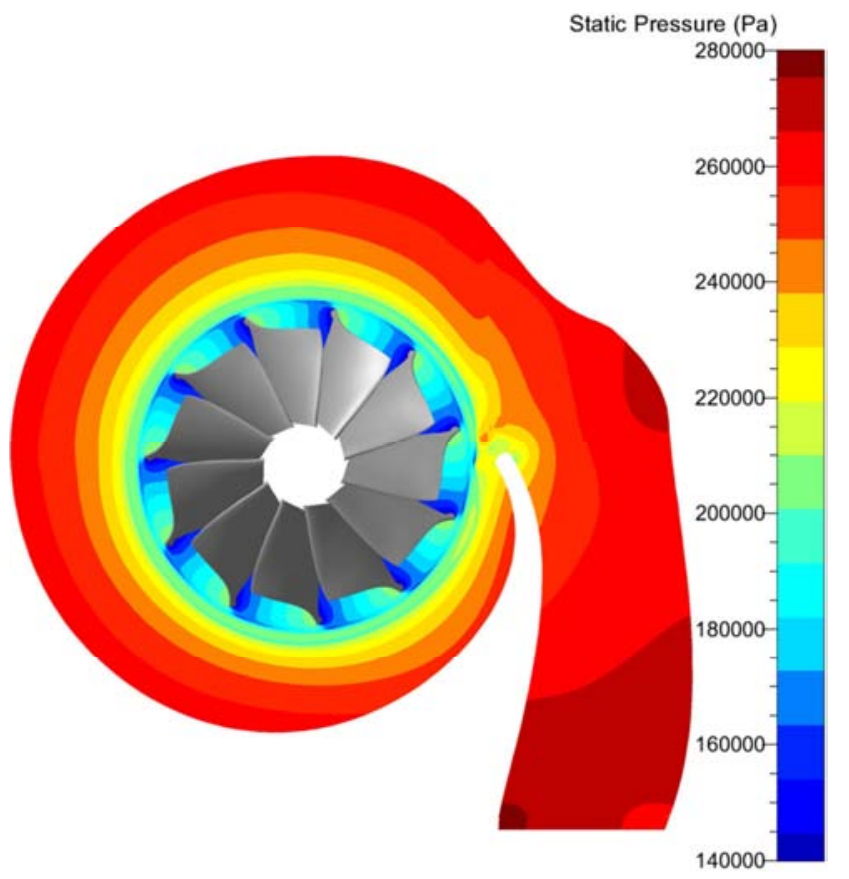

(a) Baseline.

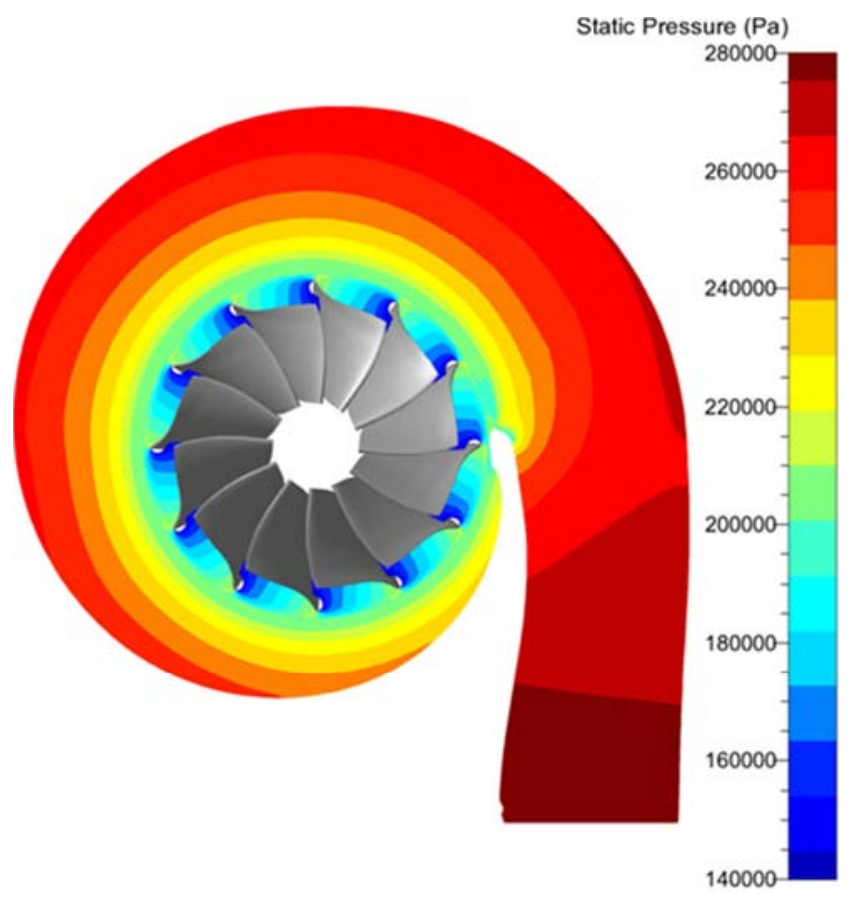

(b) Optimization.

Figure 8. Static pressure at shroud side near peak efficiency.

Because of the rapid development of modern computers, all three-dimensional flow field optimization calculations use full-period calculations to ensure calculation can represent the real turbine performance. It can be seen from Figure 8 that the static pressure flow field of the turbine near the shroud flow channel changes, the static pressure distribution in the turbine housing also changes. The turbine housing can gradually convert the internal energy of the gas into kinetic energy. The static pressure of the suction surface of the turbine at the shroud changes greatly, the overall turbine loading is larger than the original turbine. The turbine is not obviously affected by the pressure fluctuations at the tongue of the turbine housing [9].

\section{Turbine Structure Design}

The stresses of the turbine are mainly caused by the centrifugal force, thermal stress, aerodynamic force and vibration stress of the blade. The maximum stress of the turbine generally occurs at the root of the blade, where there is also a great risk of low cycle fatigue. Reliability needs to reduce the stress of the turbine to the allowable range of the life of the turbocharger. Adjusting the fillet sizes of the blade and the thickness distribution of the blade can reduce the thermal and centrifugal stress of the turbine. On the other hand, blade frequency analysis is also an important part of reliability design. Blade resonance often brings high-cycle fatigue problems [10]. The original turbine analysis showed that the original turbine has serious high-cycle fatigue problems. So it is necessary to analyze the turbine frequencies at different modes. After the process, a harmonic response analysis is also needed, and the entire turbine responds to the vibration generated by the fluctuation of the airflow through the tongue of the turbine housing. 


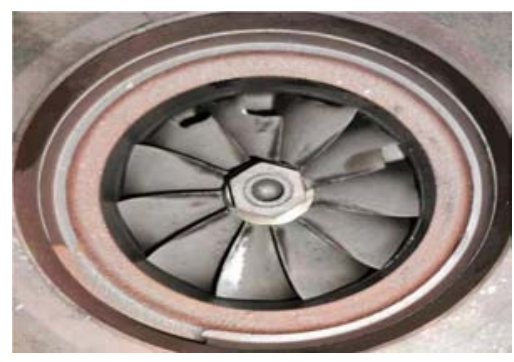

Figure 9. Original design.

This article only discusses the mechanical stress of the turbine. In the process of turbine stress analysis, first of all, the temperature field of the turbine needs to be obtained through CFD calculation. The temperature boundary to the solid model of the turbine transfers to the thermo-structure analysis. The mechanical stress analysis boundary conditions mainly consider the thermal load at the calibration speed of the engine because at this engine speed the temperature is close to max engine temperature. The rotating centrifugal force generated by the turbine operating. The stress analysis considers the maximum turbine rotating speed.

The K418 high temperature resistant material is used for the turbine material. In order to reduce the calculation time and with certain accuracy of the calculation, only a single-cycle model is used in the structure calculations. The mesh uses a full hexahedron solid45 type mesh and contains 16,100 elements for a single blade pie of the calculation. The simulation results are shown in Figure 10. It can be seen that the maximum stress of the turbine occurs at the fillet position of the suction surface, and the stress at this place is reduced by increasing the fillet size until the stresses meet the design standard.

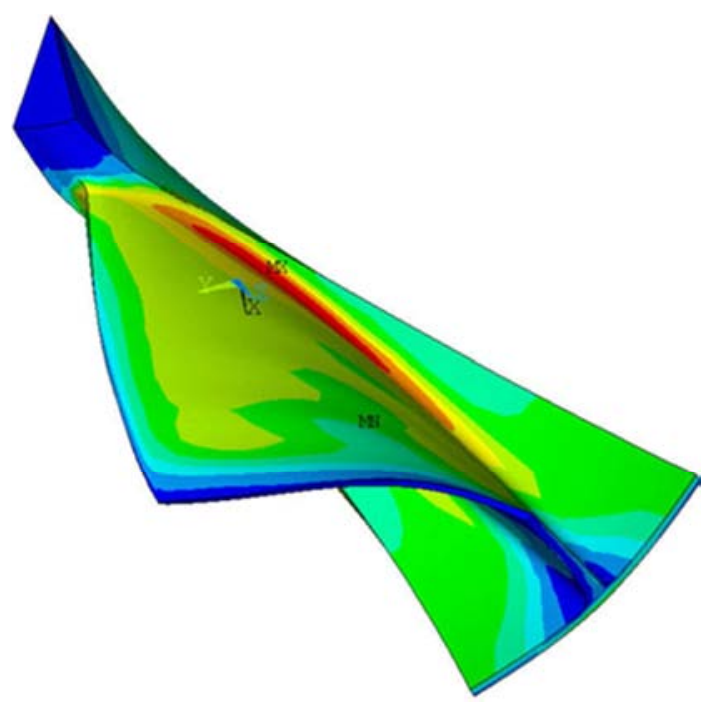

Figure 10. Turbine stress.

\section{Turbine Aerodynamic Performance Test}

In order to verify the accuracy of the turbine design, a three-dimensional printing process was used to make a test turbine wheel and housing. A turbine performance test was performed on the turbocharger performance bench. Turbine performance bench arrangement is shown in Figure 11. In order to ensure the accuracy of temperature, thermal insulation is installed on the surface of the turbocharger and the intake and exhaust pipes. The turbine inlet is connected to the combustion chamber outlet. The turbine outlet pipe is piping back to combustion inlet to form a close-loop testing system. Three pressure transducers and temperature sensors are used for pressure and temperature measurements. The compressor is installed on the other side of the turbine shaft. The flow meter is installed on the inlet side of the compressor to measure the mass flow of the compressor.

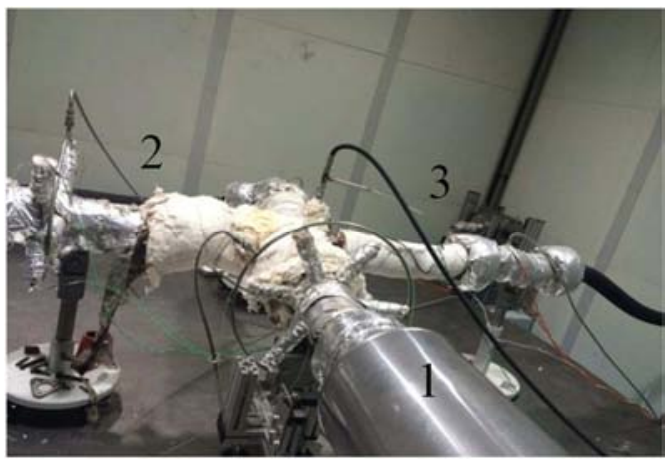

1. Burner 2. Turbine Outlet 3. Compressor inlet.

Figure 11. Turbocharger rig.

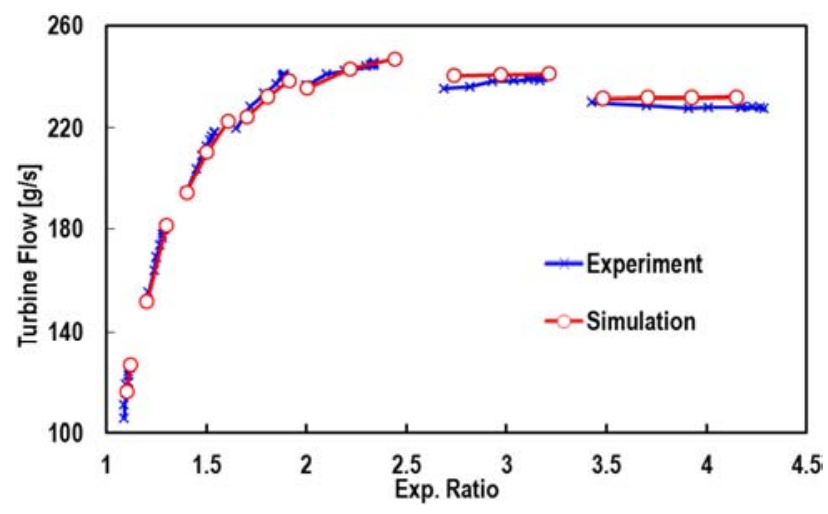

(a) Turbine flow.

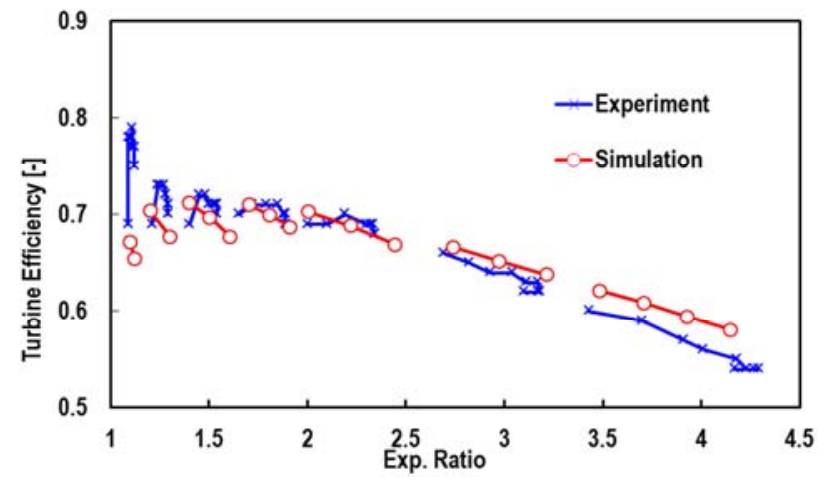

(b) Turbine efficiency.

Figure 12. Turbine performance. 
It can be seen from the turbine flow characteristic diagram in Figure 12a that the flow vs. expansion ratio characteristics is consistent with the simulation results. The large differences of the mass flow rates between tests and computations are the high-speed. The largest difference is about $2 \%$. The flow differences between tests and the simulations are small. The differences are less than $1 \%$. The turbine efficiency plot is shown in Figure 12b. It can be seen that the trend of the test and simulation results is the same. The differences are caused by the heat transfer loss and the mechanical efficiency of the bearing. The bearing loss impact is small at high speed and at is large low speed. Based on test results, we can see that the design results meet the requirements of the aerodynamic targets, and the efficiency of the original turbine is increased by $2 \%$ when the expansion ratio is 2.0 .

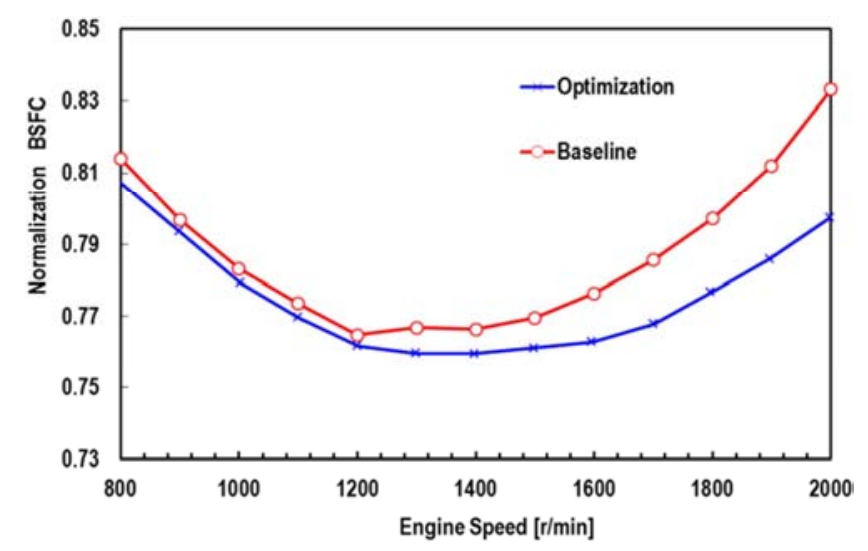

Figure 13. Engine experiment data.

The prototype was tested on a $9 \mathrm{~L}$ engine and compared with the original turbine test results as shown in Figure 13. The test boundary conditions were at sea level atmospheric environment, the engine inlet temperature was controlled at $25^{\circ} \mathrm{C}$. The air compressor was used to control the calibration point of the engines. The outlet temperature of the inter cooler is $55^{\circ} \mathrm{C}$, The other operating conditions, the intercooler outlet temperature changes with the engine flow rate. During the engine test, the exhaust back pressure is controlled to about $10 \mathrm{kPa}$. The results show that the performance of the optimized turbine is significantly improved than the original turbine. The specific fuel consumption at calibration point has been reduced by $3 \%$ (the engine speed at $2000 \mathrm{rpm}$ ).

\section{Conclusions and Discussion}

The development of modern simulation technology has driven the development of automotive turbocharger applications. In this paper, engine simulation calculations, CFD optimization, and structure analysis have been applied to the turbine design process. This paper develop a turbocharger turbine for a 9L Tier IV engine application and finally achieves the design goals both performance and reliability.

1. Through the engine simulation analysis for the original matched turbocharger, the performance gaps were found, and the aerodynamic target of the turbine design was set.

2. By scaling the existing high-efficiency in-house turbine, the initial turbine geometry was quickly obtained, and the geometry was optimized using CFD simulation to finally achieve the turbine aerodynamic goals. The new turbine efficiency improvement is more than $2 \%$ compared to the original turbine.

3. The reliability of the turbine geometry is checked to achieve the goal of stress and frequency meeting the reliability goal of the turbocharger.

4. The advanced 3D printing technology was used to rapidly manufacture the prototype of the turbine. The final turbine performances were checked on engine test. The fuel consumption of the new turbine was $3 \%$ better than the original turbine.

\section{References}

[1] Wallace, F. J., "Theoretical Assessment of the Performance Characteristics of Inward Radial Flow Turbines," Proc. ImechE, Vol. 172, No. 33, 1959.

[2] N. C. Baines and F. J. Wallace, "Computer Aided Design of Mixed Flow Turbines for Turbochargers” 1979.

[3] C. Xu, and R. S. Amano, On the Development of Turbomachine Blade Aerodynamic Design System, International Journal for Computational Methods in Engineering Science and Mech, Vol. 10, No. 3, pp. 186-196, 2009.

[4] C. Xu, (2007), "Design Experience and Considerations for Centrifugal Compressor Development," Pro Inst. Mech Eng. Part G: J. Aerosp. Eng., 221 (2), pp. 273-287.

[5] C. Xu, and R. S. Amano, "Empirical Design Considerations for Industrial Centrifugal Compressors," Int. J. Rotating Mach., 2012, p. 184061.

[6] Gong Jinke, Chen Changyou, Hu Liaoping, Yang Di, Liu Guanlin. "Computational Research on Turbocharger Matching of Electronically Controlled Bypass Valve." Journal of Hunan University: Natural Science Vol. 8: 1-7.

[7] Chen, H \& Hakeem, Irfan. (1996). "Modeling of a turbocharger turbine under pulsating inlet conditions". Pro Inst. Mech Eng. Part A: Journal of Power and Energy, Vol. 2010. A04695.

[8] Xu, C. \& Amano, Ryo. (2017). "Effects of Asymmetric Radial Clearance on Performance of a Centrifugal Compressor". ASME, Journal of Energy Resources Technology 140 (5), NOV, 2017. DOI: $10.1115 / 1.4038387$.

[9] Ghenaiet, Adel \& Mahfoudh, Cerdoun. (2014). "Simulations of Steady and Unsteady Flows Through a Twin-Entry Radial Turbine". Proceedings of the ASME Turbo Expo. 2. 10.1115/GT2014-25764.

[10] Aschenbruck, J., \& Seume, J. R. (2015). "Experimentally verified study of regeneration-induced forced response in axial turbines". Journal of turbomachinery, 137 (3), 031006.1-031006.10. 